\title{
Role of meteorological conditions in reported chickenpox cases in Wuhan and Hong Kong, China
}

\author{
Banghua Chen ${ }^{1}$, Ayako Sumi ${ }^{2^{*}}$ D, Lei Wang ${ }^{3}$, Wang Zhou ${ }^{4 *}$ and Nobumichi Kobayashi ${ }^{2}$
}

\begin{abstract}
Background: Chickenpox is a common contagious disease that remains an important public health issue worldwide. Over $90 \%$ of unvaccinated individuals become infected, but infection occurs at different ages in different parts of the world. Many people have been infected by 20 to 30 years of age in China, and adults and pregnant women who become infected often develop severe infection. Furthermore, a mortality rate of 2-3 per 100,000 infected persons has been reported. In this study, we explore the temperature-dependent transition of patterns of reported chickenpox cases in two large subtropical climate cities, Wuhan and Hong Kong, China, to aid in the prediction of epidemics and preparation for the effects of climatic changes on epidemiology of chickenpox in China.
\end{abstract}

Methods: We used a time series analysis comprising a spectral analysis based on the maximum entropy method in the frequency domain and the nonlinear least squares method in the time domain. Specifically, the following time series data were analyzed: data of reported chickenpox cases and meteorological data, including the mean temperature, relative humidity and total rainfall in Wuhan and Hong Kong from January 2008 to June 2015.

Results: The time series data of chickenpox for both Wuhan and Hong Kong have two peaks per year, one in winter and another in spring, indicating a bimodal cycle. To investigate the source of the bimodal cycle of the chickenpox data, we defined the contribution ratio of the 1-year cycle, $Q_{1}$, and the 6-month cycle, $Q_{2}$, as the contribution of the amplitude of a 1-year cycle and a 6-month cycle, respectively, to the entire amplitude of the time-series data. The $Q_{1}$ values of Wuhan and Hong Kong were positively correlated with the annual mean temperature and rainfall of each city. Conversely, the $Q_{2}$ values of Wuhan and Hong Kong were negatively correlated with the annual mean temperature and rainfall of Wuhan and Hong Kong.

Conclusion: Our results showed that the mean temperature and rainfall have a significant influence on the incidence of chickenpox, and might be important predictors of chickenpox incidence in Wuhan and Hong Kong.

Keywords: Chickenpox, Seasonality, Temperature, Rainfall, Time-series analysis

\section{Background}

Chickenpox (varicella), which is caused by the ubiquitous varicella-zoster virus, is an extremely contagious vaccine-preventable infectious disease [1-4]. Over $90 \%$ of unvaccinated individuals become infected, but infection occurs at different ages in different parts of the world. For example, over $80 \%$ of people have been

\footnotetext{
* Correspondence: sumi@sapmed.ac.jp; rising-up@hotmail.com

${ }^{2}$ Department of Hygiene, Sapporo Medical University School of Medicine,

S-1, W-17, Chuo-ku, Sapporo, Hokkaido 060-8556, Japan

${ }^{4}$ Wuhan Centers for Disease Control and Prevention, 24 Jianghanbei Road,

Wuhan, Hubei 430000, China

Full list of author information is available at the end of the article
}

infected by 10 years of age in the United States, the United Kingdom, and Japan, while many people have been infected by 20 to 30 years of age in China, India, Southeast Asia, and the West Indies [5]. In these areas, adults and pregnant women who become infected often develop severe infection. Furthermore, a mortality rate of 2-3 per 100,000 infected persons has been reported [6]. Therefore, chickenpox remains an important public health issue, especially in China, India, Southeast Asia, and the West Indies.

Potential transmission of infectious diseases including chickenpox is believed to be affected by changes in 
climate, and some studies have examined the relationship between weather variability and the incidence of infectious diseases $[4,7,8]$. We previously demonstrated that temperature was associated with reported chickenpox incidence in Japan [3]. Therein, bimodal cycles of reported chickenpox incidences that were clearly observed in northern Japan disappeared at lower latitudes, and unimodal cycles appeared in southern Japan. This transition of patterns of reported chickenpox incidences in Japan was considered to be temperaturedependent.

The present study was conducted to explore whether the temperature-dependent transition of patterns of reported chickenpox incidences in Japan is applicable to areas in which chickenpox incidences are a serious problem, such as China, India, Southeast Asia, and the West Indies. In the present study, we investigated the seasonality of reported chickenpox cases in Hong Kong and Wuhan, China, where a nationwide internet-based infectious diseases reporting system has been established and accumulated good-quality surveillance data on chickenpox [9]. The analyses included spectral analyses conducted using the maximum entropy method (MEM) and the least squares method (LSM) $[3,8]$. The results presented herein will facilitate prediction of epidemics and preparation for the effects of climatic changes on the epidemiology of chickenpox in China.

\section{Methods}

\section{Data}

\section{Chickenpox data}

Chickenpox is considered an easily diagnosed childhood disease due to the presence of an often pathognomonic rash; therefore, it is rarely subject to misdiagnosis and miscoding [10, 11]. Case reports of chickenpox in Wuhan and Hong Kong were defined by medical doctors as acute generalized maculopapular vesicular rashes without other apparent cause $[4,12]$.

\section{Wuhan}

Wuhan has an area of $8494 \mathrm{~km}^{2}$ and a population of 10 million (as of 2015) [13]. As of 2015, the age distribution of the Wuhan population was as follows: infants and preschool children, aged $\leq 4$ years $(3.6 \%)$; pre-teens, aged 5-9 years (3.8\%); teens, aged $10-19$ years $(10.2 \%)$; and adults, aged $\geq 20$ years $(82.4 \%)$. Though chickenpox was not one of the notifiable infectious diseases in China, it was categorized as a surveillance infectious disease by the Wuhan Department of Health in 2006, after which hospitals in Wuhan were required to report chickenpox cases on-line to the China Information System for Disease Control and Prevention using a standardized form. In this study, we analyzed the weekly number of cases of chickenpox reported in Wuhan between January
2008 and June 2015 (390 data points). The data are available from the CISDCP website through the Wuhan Centers for Disease Control and Prevention.

\section{Hong Kong}

Hong Kong has an area of $1104 \mathrm{~km}^{2}$ [13] and a population of 7.3 million (as of 2015) [14]. As of 2015, the age distribution of the Hong Kong population was as follows: infants and preschool children, aged $\leq 4$ years (3.8\%); pre-teens aged 5-9 years (4.0\%); teens, aged 10 19 years (8.3\%); and adults, aged $\geq 20$ years $(83.9 \%)$ [14]. In Hong Kong, China, chickenpox is a notifiable infectious disease; therefore, all medical practitioners in both public and private sectors are required to report all cases to the Centre of Health Protection (CHP), Department of Health, Hong Kong Special Administrative Region Government via a centralized notification system. The monthly number of chickenpox cases were obtained from the CHP for January 2008 to June 2015 (90data points) (http://www.chp.gov.hk).

\section{Meteorological data}

Using daily temperature, relative humidity, rainfall, and wind velocity data for 2008-15 for Wuhan and for Hong Kong [15], we calculated mean values, which corresponded to average daily data (one data point). We also calculated the total daily rainfall for the two places for 2008-15 (one data point).

\section{Wuhan}

Wuhan is located in a subtropical wet monsoon climate area in which there is heavy rainfall and four clearly defined seasons. Seasons were defined as spring (April), summer (May-September), autumn (October) and winter (November-March). Daily meteorological data, including the mean temperature, relative humidity and total rainfall, were collected in the study region by the Meteorological Department, Wuhan, which received and managed real-time data from 116 meteorological surveillance sites widely distributed in Wuhan. The daily data were gathered for a total of 2922 days from January 2008 to June 2015 (2922 data points).

\section{Hong Kong}

Hong Kong is located in eastern Asia, bordering the South China Sea to the south, west, and east, and mainland China to the north. It has a subtropical and monsoon-influenced climate with wet and hot summers and dry and cool to mild winters [8]. Seasons are defined as spring (March-May), summer (June-August), autumn (September-November), and winter (December-February). Meteorological data including daily mean temperature, mean relative humidity and rainfall were obtained from January 2008 to June 2015 from daily meteorological 
reports from the Hong Kong Observatory website [15]. We obtained daily data for a total of 2922 days from January 2008 to June 2015 (2922 data points).

\section{Vaccine}

Chickenpox vaccine became available in Wuhan in 2006. Parents wishing to have their children vaccinated must pay for the service. The estimated chickenpox vaccination rate was less than $10 \%$ in children aged under 6 years, according to vaccine information from Wuhan Central Disease Control.

In Hong Kong, chickenpox vaccines have been incorporated in the region's universal Childhood Immunisation Programme as a free, optional vaccine since July 2014 for children born on or after January 1, 2013. Children are supposed to receive two doses of the vaccine. The first dose is given at age 1 year. The second dose is given when the children enter the first year of primary school [16]. Before the implementation of universal varicella vaccination, children in Hong Kong were able to receive chickenpox vaccination only through the private sector. A survey conducted by Hong Kong's Department of Health reported that only $32.4 \%$ of locally born children had received varicella vaccine in 2009 [17]. A 2012 survey of students aged 2-6 years in Hong Kong kindergartens revealed that the varicella vaccination rate was $57.6 \%$ [18]. Although varicella vaccine coverage has increased in Hong Kong, it remains below that of the United States (79.9\%-92.0\%) [19].

\section{Data analysis}

The effects of meteorological conditions (temperature, relative humidity, rainfall) on the reported number of chickenpox cases were estimated as previously described [3]. An outline of the analysis procedure is as follows.

(1)Setting up time-series data for the analysis: equal sampling time intervals of the original time series data $x(t)$ (where $t=$ time) are chosen, lack of $x(t)$ compensated for, outliers corrected, logarithm transformation performed, and removal of long-term trend of $x(t)$ performed, if necessary.

(2) MEM spectral analysis: spectral analysis in the frequency domain is conducted based on the maximum entropy method (MEM), and the power spectral density (PSD) is obtained. From the result of PSD, periodic modes constructing seasonality of $x(t)$ are assigned. The formulation of MEM-PSD is described in the reference [8].

(3) Least squares method (LSM): the validity of the MEM spectral analysis results was confirmed by calculation of the least squares fitting (LSF) curve to $x(t)$ with MEM estimated periods. The formulation of the LSF curve in $X(t)$ is described as follows:

$$
X(t)=A_{0}+\sum_{n=1}^{N} A_{n} \cos \left\{2 \pi f_{n}\left(t+\theta_{n}\right)\right\}
$$

which is calculated using the LSM for $x(t)$ with unknown parameters $f_{n}, A_{0}$ and $A_{\mathrm{n}}(n=1,2,3, \ldots, N)$, where $f_{n}$ $\left(=1 / T_{n} ; T_{n}\right.$ is the period) is the frequency of the $n$-th component, $A_{0}$ is a constant that indicates the average value of the time series data, $A_{n}$ and $\theta_{n}$ are the amplitude and the phase of the $n$-th component, respectively, and $N$ is the total number of components.

(4) Contribution ratio: a 'contribution ratio' was defined for assignment of periodic modes constructing the seasonality of the original time series data $x(t)$ [20-24]. The contribution ratio $Q_{n}$ is described as follows:

$$
Q_{n}=A_{n}^{2} / Q
$$

where, $A_{n}$ indicates the amplitude of the $n$-th periodic mode constituting the LSF curve $X(t)$ to the original data $x(t)$ [Eq. (1)], and $Q$ is the total power of $x(t)$.

\section{Results \\ Age distribution}

From January 2008 to June 2015, 32,671 cases and 72,295 cases of chickenpox were reported in Wuhan and Hong Kong, respectively. The age distribution of the reported cases in Wuhan is shown in Fig. 1. In Wuhan, chickenpox cases mainly affected schoolchildren and adolescents (5-19 years). During the study period, 32\%$42 \%$ and $23 \%-32 \%$, respectively, of all cases were reported in individuals aged 5-9 and 10-19 years. Also in Wuhan, 18,815 (58\%) male and 13,856 (42\%) female cases of chickenpox were reported. We did not have access to age and sex distribution data concerning reported cases of chickenpox in Hong Kong.

\section{Temporal variations in reported chickenpox incidence}

Figure 2 indicates the weekly reported chickenpox incidence data for Wuhan (a) and Hong Kong (b). A 1-year chickenpox data cycle is shown for each location. For Wuhan (Fig. 2a), two peaks, one in winter and another in spring, were superimposed on a 1-year cycle. This bimodal cycle was clearly observed in the case of Hong Kong (Fig. 2b).

\section{Spectral analysis and LSF analysis}

The PSDs based on the MEM for chickenpox data (Fig. 2) are shown in Fig. 3. In each PSD, the prominent spectral line was observed at $f=1.0[f(1 /$ year $)$; frequency $]\left(=f_{1}\right)$ corresponding to the 1 -year cycle, and the spectral lines of $f_{2}$ 


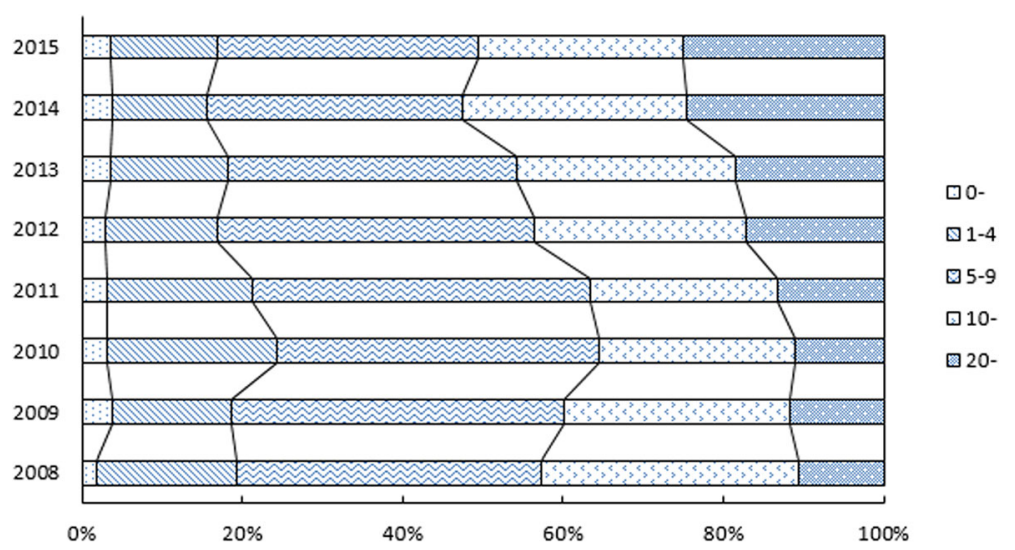

Fig. 1 Age distribution of reported chickenpox cases in Wuhan
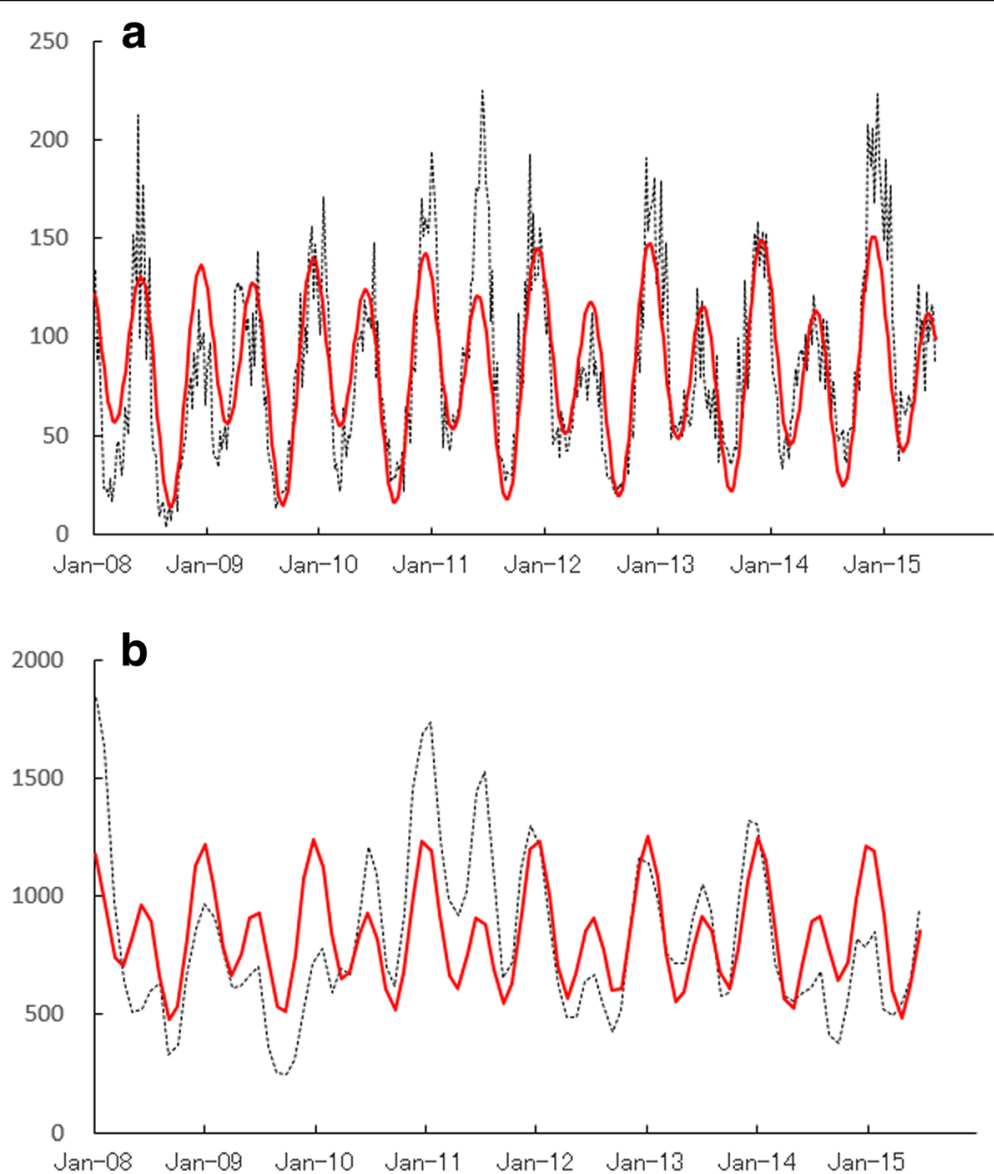

Time (year)

Fig. 2 Comparison of least-squares fitting curves calculated for 1.0- and 0.5-year periodic modes (red solid line) with original data (black dotted line) from January 2008 to June 2015 for: a Wuhan (weekly data), b Hong Kong (monthly data) 


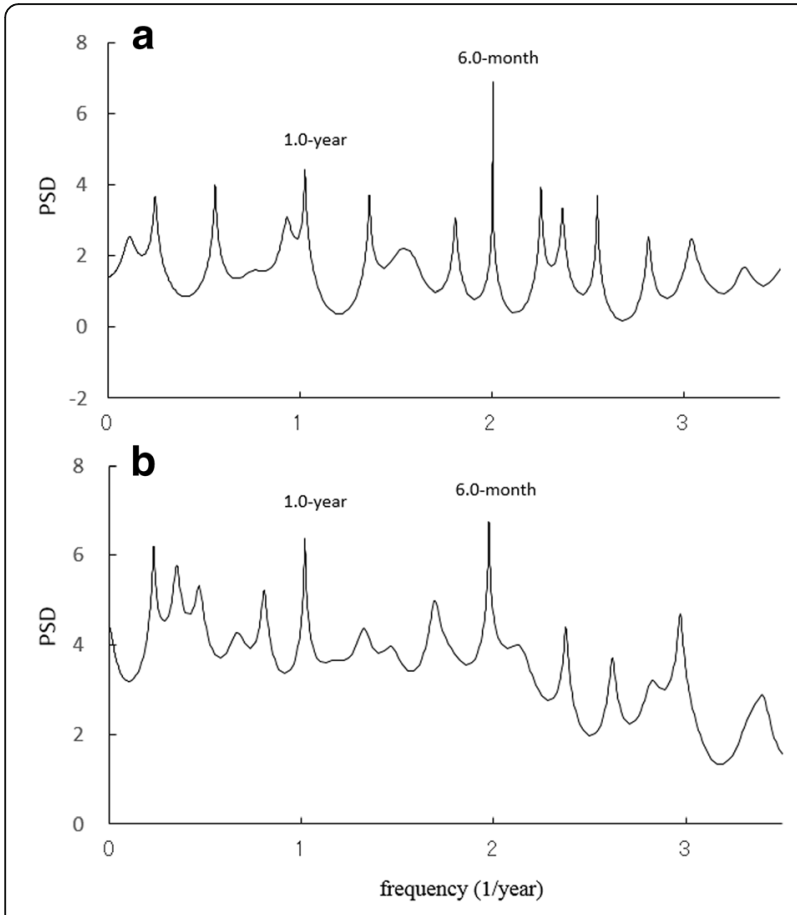

Fig. 3 Power spectral density of the original data for: a Wuhan, b Hong Kong

$\left(=f_{1} \times 2\right)$ corresponding to the 6 month cycle were observed at $f=2.0$. To investigate the seasonality of each set of chickenpox data (Fig. 2) in detail, the LSF curve in Eq. (1) was calculated for the 1-year and 6-month cycles that were clearly observed in the PSD (Fig. 3). The LSF curves are shown in Fig. 2. In the figure, both LSF curves demonstrated a bi-modal seasonal cycle with the first peak in winter (December-January) and the second peak in spring (May-July). The contribution ratios [Eq. (2)] of the 1-year cycle $\left(\mathrm{Q}_{1}\right)$ for Wuhan and Hong Kong were 0.13 and 0.21 , respectively. The contribution ratios of the 6-month cycle $\left(\mathrm{Q}_{2}\right)$ for Wuhan and Hong Kong were 0.65 and 0.39, respectively. The summation of the $Q_{1}$ and $Q_{2}$ values for Wuhan and Hong Kong were 0.78 (corresponding to 78\%) and 0.60 (corresponding to 60\%), respectively, indicating that these cycles made major contributions to the seasonal oscillations.

\section{Correlation between reported chickenpox incidences and meteorological conditions}

Figure $4 a-c$ and Fig. $4 a^{\prime}-c^{\prime}$ show plots of the contribution ratio of the 1-year cycle $\left(Q_{1}\right)$ and the 6-month cycle $\left(Q_{2}\right)$, respectively, to the mean temperature and relative humidity and a summation of the rainfall data for January 2008-June 2015. These figures also show results reported for Japan in a previous study [3]. The meteorological data for Wuhan and Hong Kong presented in Fig. 4 are listed in Table $1 a, b, d$.

\section{Temperature}

As shown in Fig. $4 a$, the $Q_{1}$ values of Wuhan and Hong Kong were positively correlated with the mean annual temperatures of Wuhan $\left(16.9{ }^{\circ} \mathrm{C}\right)$ and Hong Kong $\left(23.2{ }^{\circ} \mathrm{C}\right)$. This trend was essentially the same as for Japan (Fig. 4a), where the value of $Q_{1}$ increased as the annual mean temperature increased. The $Q_{1}$ values for Wuhan and Hong Kong were smaller than those for prefectures in Japan in the same yearly mean temperature range.

As shown in Fig. 4a, the $Q_{2}$ values of Wuhan and Hong Kong were negatively correlated with the annual mean temperature of Wuhan $\left(16.9^{\circ} \mathrm{C}\right)$ and Hong Kong $\left(23.2^{\circ} \mathrm{C}\right)$. This trend is essentially same as that observed in Japan (Fig. 4a), where the $Q_{2}$ value decreased as the annual mean temperature increased. The $Q_{2}$ values for Wuhan and Hong Kong were larger than those for prefectures in Japan in the same yearly mean temperature range.

\section{Relative humidity}

For Wuhan and Hong Kong, the $Q_{1}$ (Fig. $4 b$ ) and $Q_{2}$ (Fig. $4 b^{\prime}$ ) values were correlated with the annual mean relative humidity when it ranged from $76.4 \%$ to $78.3 \%$, while the $Q_{1}$ and $Q_{2}$ values for Japan were correlated with relative humidity of $59.3 \%$ to $77.2 \%$ (Figs. $4 b$ and b'). No clear trends in variations of $Q_{1}$ and $Q_{2}$ were observed for Wuhan and Hong Kong.

\section{Rainfall}

For the annual mean rainfall (Fig. 4c), the $Q_{1}$ values of Wuhan and Hong Kong were positively correlated with the annual mean rainfall of Wuhan $(1798 \mathrm{~mm})$ and Hong Kong $(2364 \mathrm{~mm})$. This trend was essentially the same as observed for Japan (Fig. 4c), where the value of $Q_{1}$ increased as the annual mean rainfall increased. The values of $Q_{1}$ for Wuhan and Hong Kong were smaller than those for prefectures in Japan in the same yearly mean rainfall range.

For the annual mean rainfall (Fig. $4 c^{\prime}$ ), the values of $Q_{2}$ of Wuhan and Hong Kong were negatively correlated with the annual mean rainfall of Wuhan $(1798 \mathrm{~mm})$ and Hong Kong $(2364 \mathrm{~mm})$. This trend is essentially the same as that observed for Japan (Fig. 4c), where the $Q_{2}$ value decreased as the annual mean rainfall increased. The $Q_{2}$ values for Wuhan and Hong Kong were larger than those for prefectures in Japan in the same yearly mean rainfall range.

\section{Discussion}

The results of the present study revealed that the $Q_{1}$ and $Q_{2}$ of temperatures for Wuhan and Hong Kong (Figs. 4a and a') were essentially same as for Japan. In our preceding study, bimodal cycles of reported chickenpox incidences that were clearly observed in areas of northern 


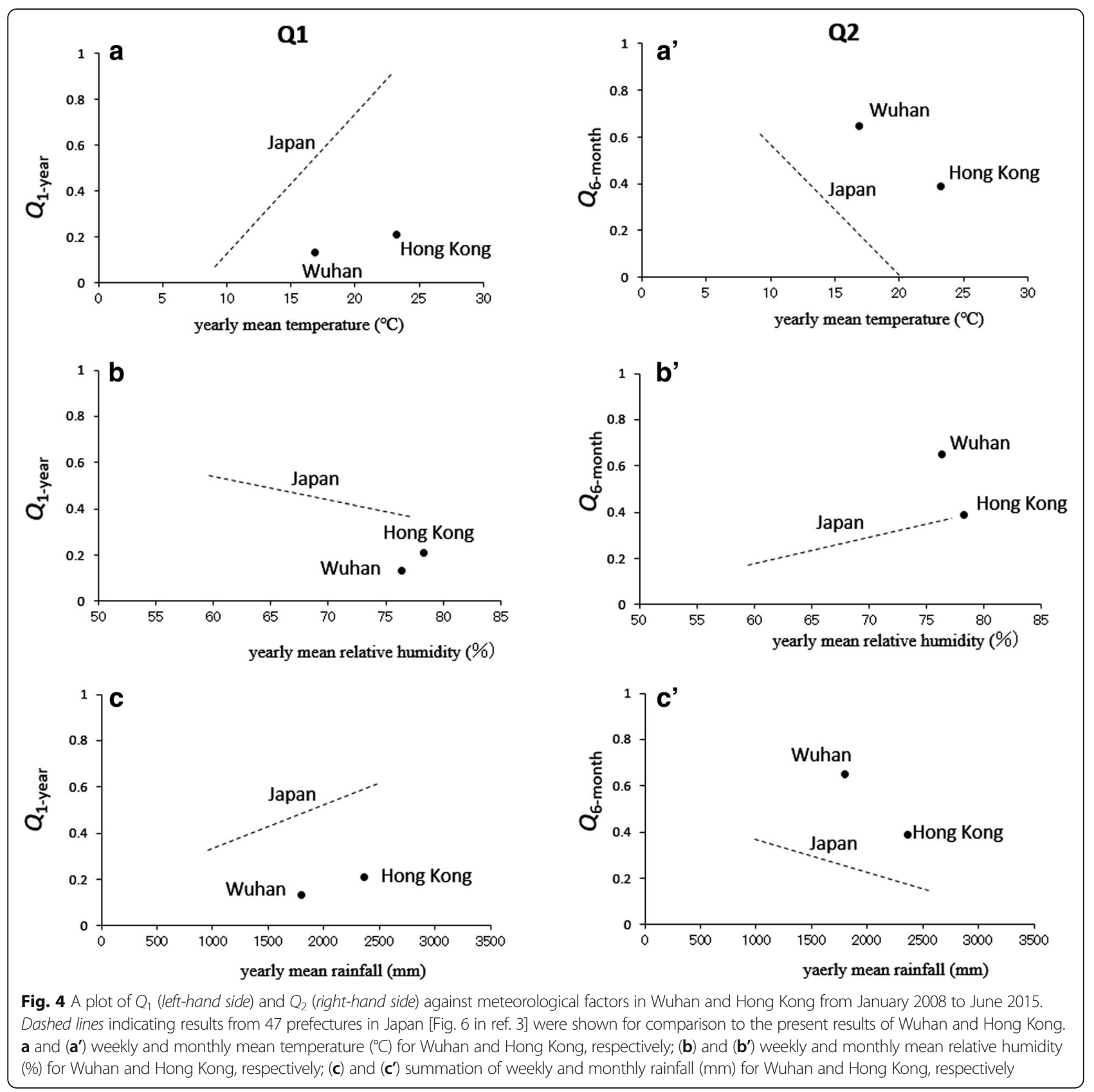

Table 1 The value of mean, standard deviation (SD) and SD/mean for daily temperature (a), daily relative humidity (b), daily rainfall (c), and (d) the value of summation of the daily rainfall during 2008-2015 for Wuhan and Hong Kong

\begin{tabular}{|c|c|c|c|c|c|c|c|c|c|c|}
\hline & \multicolumn{2}{|c|}{ a. Daily temperature } & \multirow[b]{2}{*}{$\mathrm{SD} / \mathrm{mean}$} & \multicolumn{2}{|c|}{ b. Daily relative humidity } & \multirow[b]{2}{*}{$\mathrm{SD} / \mathrm{mean}$} & \multicolumn{2}{|c|}{ c. Daily rainfall } & \multirow[b]{2}{*}{$\mathrm{SD} /$ mean } & \multirow{2}{*}{$\begin{array}{l}\text { d. Summation of the } \\
\text { daily rainfall }\end{array}$} \\
\hline & mean $\left({ }^{\circ} \mathrm{C}\right)$ & $\mathrm{SD}\left({ }^{\circ} \mathrm{C}\right)$ & & mean $(\%)$ & SD (\%) & & mean $(\mathrm{mm})$ & $\mathrm{SD}(\mathrm{mm})$ & & \\
\hline Wuhan & 18.9 & 9.2 & 0.49 & 76.4 & 8.6 & 0.11 & 34.9 & 116.2 & 3.33 & 1797.8 \\
\hline Hong Kong & 23.2 & 4.9 & 0.21 & 78.3 & 6.3 & 0.08 & 6.4 & 20.0 & 3.12 & 2363.8 \\
\hline
\end{tabular}


Japan such as Hokkaido (latitude $43^{\circ} \mathrm{N}$ ) disappeared at lower latitudes, and unimodal cycles appeared in Okinawa Prefecture, the most southern prefecture in Japan (latitude $26^{\circ} \mathrm{N}$ ). This transition of patterns of reported chickenpox incidences in Japan was considered to be temperaturedependent. Thus, it is reasonable that temporal patterns of chickenpox incidences observed for Wuhan and Hong Kong (Fig. 2) were dominated by temperature as well.

The trends of $Q_{1}$ and $Q_{2}$ of temperature for Wuhan and Hong Kong (Fig. 4a and a') support the findings reported by Shoji et al. [25], who showed that the reported cases of chickenpox increased at $5{ }^{\circ} \mathrm{C}-20{ }^{\circ} \mathrm{C}$ (i.e., the temperature range at which the chickenpox virus is activated) and decreased at temperatures lower than $5{ }^{\circ} \mathrm{C}$ and higher than $20{ }^{\circ} \mathrm{C}$. In Wuhan, where the temperature falls below $5{ }^{\circ} \mathrm{C}$ in winter and exceeds $20^{\circ} \mathrm{C}$ in summer, the occurrence of epidemics is bimodal (Fig. 2a). In contrast, the occurrence of epidemics is expected to be unimodal in Hong Kong, where the temperature rarely falls below $5{ }^{\circ} \mathrm{C}$ in winter; however, it was actually bimodal (Fig. 2b). This bimodal cycle of chickenpox epidemics in Hong Kong may be related to the fact that the values of $Q_{2}$ for Hong Kong are larger than those for Japanese prefectures with the same mean temperature (Fig. 4a'). One study revealed a spring peak in a bimodal pattern of chickenpox cases related to spring vacation [26]. With respect to Wuhan and Hong Kong, school children do not have spring vacation; thus, it is unlikely that the occurrences of bimodal reported chickenpox incidences in Wuhan and Hong Kong are related to the school calendar. Rather, the bimodal pattern likely results from the mean temperature, as shown in Fig. 4a'.

The increase in the magnitude of $Q_{2}$ for Wuhan and Hong Kong (Fig. 4a') may be explained in terms of (i) the age distribution of reported chickenpox cases, as well as (ii) the effect of rainfall.

(i) Age distribution of reported chickenpox cases: For Hong Kong, more than $90 \%$ of the notifications were regarding children aged $<18$ years, with $29.1 \%$ of notified pediatric cases of chickenpox receiving treatment at public or private hospitals [27]. For Wuhan and Hong Kong, the proportion of reported cases among school children was high relative to Japan. Specifically, the 519 year age group accounted for $60 \%-70 \%$ of all cases from 2007 to 2015 for Wuhan and the 6-17 year age group accounted for $52.4 \%$ of all cases for Hong Kong [18], while pre-school children (0-4 years) accounted for $78 \%$ of the cases reported during 2009-2011 for Japan [3]. For Wuhan and Hong Kong, it can be assumed that school children tend to stay indoors during the day, which in turn increased domestic transmission. Given the limited indoor space in schools in Wuhan and Hong Kong, school children may have a greater opportunity for close contact, facilitating chickenpox transmission. In addition, the high population densities of Wuhan
(1248 persons $/ \mathrm{km}^{2}$ ) and Hong Kong (6622 persons $/ \mathrm{km}^{2}$ ) may make it easier for chickenpox to spread from one person to another.

(ii) Effect of rainfall: It has been reported that rainfall was positively associated with chickenpox notifications in Hong Kong [2]. Indeed, the annual mean summation of rainfall in Hong Kong $(2364 \mathrm{~mm})$ is larger than that of Wuhan $(1798 \mathrm{~mm})$ and 43 of the 47 prefectures in Japan (Fig. 4c'). During the hot and wet season with high temperature and heavy rain, children tend to stay indoors with air-conditioning, which in turn increases domestic transmission. Given the limited indoor space in Hong Kong and Wuhan, children may have a greater opportunity for close contacts with one another, facilitating chickenpox transmission. However, the exact mechanism for the association between rainfall and chickenpox notifications remains unclear.

Critselis et al. examined the influence of meteorological conditions on chickenpox in Greece; before introduction of the vaccine, the authors found that the occurrence of hospitalized chickenpox cases was positively associated with wind speeds of $2.7-3.5 \mathrm{~m} / \mathrm{s}$ [28]. We confirmed that for Wuhan, the $Q_{1}$ and $Q_{2}$ values were, respectively, 0.13 and 0.63 with an annual mean wind speed of $1.8 \mathrm{~m} / \mathrm{s}$; for Japan's 47 prefectures, the mean wind speed appeared to display a randomly scattered pattern (Additional file 1).

As with Japan, the $Q_{1}$ and $Q_{2}$ values for Wuhan and Hong Kong are evidently dominated by temperature [3]; the meteorological data appear in Table 1. In Table 1c, there is clearly large variance in the daily rainfall data for Wuhan and Hong Kong. The amount of rainfall depends on the amount of water vapor in the atmosphere, which influences relative humidity [29]. The variation in the relative humidity for Wuhan and Hong Kong (Table 1b) was relatively small compared with that for rainfall (Table $1 c$ ). This finding is the result of relative humidity being constrained by the amount of saturated water vapor, which is dependent on air temperature [29]. Thus, it is reasonable to infer that the unimodal and bimodal cycles observed in temporal variations of the reported chickenpox incidence were dominated by temperature.

There was a large difference between vaccine coverage in Wuhan (10\%) and Hong Kong (57.6\%); however, the temporal patterns of the incidence data for the two areas indicated approximately the same pattern, i.e., the bimodal cycle (Fig. $2 a, b$ ). Thus, the vaccination coverage rate may not have affected the temporal patterns of the incidence data during the period of the present study (2008-15). In a previous investigation, we confirmed that the bimodal cycle of chickenpox epidemics observed in Japan was independent of the influence of the vaccination coverage rate $(20 \%-40 \%)$ [3]. If the vaccination coverage rate exceeded $80 \%$ in Wuhan, Hong Kong, and 
Japan, the seasonal peak superposed on a 1-year cycle would diminish; that phenomenon has been observed in the United States [19].

It is possible that the epidemiology of chickenpox in subtropical Wuhan and Hong Kong differs from that in temperate Japan. In a previous study, we found that preschool children (aged $\leq 4$ years) in Japan accounted for over $70 \%$ of all chickenpox cases in 2000-11 [3]. In Wuhan, chickenpox typically occurs at a later age, with many cases among schoolchildren and adolescents (519 years). The relatively low number of reported chickenpox cases among preschool children ( $\leq 4$ years) in Wuhan may be the result of the reduced chickenpox virus transmission reported for tropical areas [30]. Garnett et al. [31] proposed that in tropical regions, the transmission potential of the chickenpox virus could be adversely affected by a combination of high ambient temperatures and humidity. For example, outbreaks of chickenpox in India appear to be more common in the cooler than in the warmer months of the year [32]. By contrast, in equatorial regions, such as Singapore, the incidence does not seem to vary according to the time of year [33].

Recently, Rice [34] interpreted the seasonality of the reported incidence of chickenpox in tropical regions with respect to levels of ultraviolet radiation and air pollution. Accordingly, the effect of meteorological factors on chickenpox incidence may differ from one country to another in different climate regions. To determine the underlying causes of chickenpox virus transmission in each climate region and effectively utilize the obtained results for health services, it is necessary to conduct a systematic study; the investigation should quantify the impact of meteorological factors on the chickenpox incidence in various countries in each climate region. Toward that end, the susceptible-exposed-infected-recovered (SEIR) model, which is a well-known mathematical model of infectious disease epidemics, may be appropriate; it has been shown to be effective with measles [35]. Thus, we would expect that a theoretical procedure, such as the SEIR model, could contribute to future investigations into the meteorological factors related to chickenpox transmission [35].

It is important to assess the sensitivity and representativeness of incidence data, as was performed by Souty et al. [36] for chickenpox in France. However, the CISDCP in Wuhan and CHP in Hong Kong have not announced the relevant information (such as age, location, and number of reported severe cases of chickenpox) that would allow us to determine the sensitivity and representativeness of the data used in the present study. There should be increased awareness of the information needed to determine the sensitivity and representativeness of the data in the surveillance system; that would allow a more accurate estimate of the burden of chickenpox and help prevent it in Wuhan and Hong Kong.

It should be noted that this study was limited in that, for Hong Kong, we used monthly data for chickenpox, while we used weekly data for Wuhan, because monthly measures are the minimum unit of measurement released by the Hong Kong Observatory website. We investigated the $Q_{1}$ and $Q_{2}$ values for the monthly data for Wuhan, by converting the weekly data for Wuhan into monthly data to enable comparisons with the results shown in Fig. 4. Using the monthly data, we confirmed that the Q1 and Q2 values (0.14 and 0.68, respectively) were essentially consistent with the weekly data $(0.13$ and 0.65 , respectively). Thus, we concluded that the effect on the results of using monthly data compared with weekly data for Wuhan was negligible (Additional file 2). Further studies using weekly data for Hong Kong should be conducted to address the issue of the relationship between climate patterns and chickenpox epidemiology.

\section{Conclusion}

We confirmed that mean temperature and rainfall have a significant influence on the incidence of chickenpox, and that these factors might be important predictors of chickenpox incidence in Wuhan and Hong Kong. Further time-series analyses of chickenpox and meteorological data from other regions in China may help determine the disease's potential relationship to epidemiological factors.

\section{Additional files}

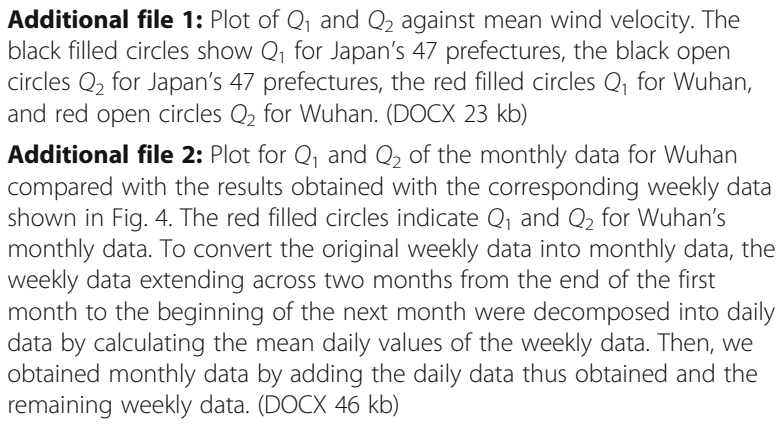

Additional file 1: Plot of $Q_{1}$ and $Q_{2}$ against mean wind velocity. The black filled circles show $Q_{1}$ for Japan's 47 prefectures, the black open circles $Q_{2}$ for Japan's 47 prefectures, the red filled circles $Q_{1}$ for Wuhan, and red open circles $Q_{2}$ for Wuhan. (DOCX 23 kb)

Additional file 2: Plot for $Q_{1}$ and $Q_{2}$ of the monthly data for Wuhan compared with the results obtained with the corresponding weekly data shown in Fig. 4. The red filled circles indicate $Q_{1}$ and $Q_{2}$ for Wuhan's monthly data. To convert the original weekly data into monthly data, the weekly data extending across two months from the end of the first month to the beginning of the next month were decomposed into daily data by calculating the mean daily values of the weekly data. Then, we obtained monthly data by adding the daily data thus obtained and the remaining weekly data. (DOCX $46 \mathrm{~kb}$ )

\section{Abbreviations \\ CISDCP: China information system for disease control and prevention; LSF: Least squares fitting; LSM: Least squares method; MEM: Maximum entropy method; PSD: Power spectral density}

\section{Acknowledgements}

The authors thank Edanz Group Ltd. for editorial assistance.

\section{Funding}

This study was supported in part by JSPS KAKENHI grant no. JP16K09061, JP25460769, JP25305022. 


\section{Availability of data and materials}

The dataset of Hong Kong analyzed during the current study is available at http://www.chp.gov.hk/en/notifiable1/10/26/43.html.

\section{Authors' contributions}

BC conceived the study and managed and analyzed the chickenpox incidence data. Data collection was administered and supervised by LW and WZ. AS analyzed the data and drafted the manuscript. NK attracted funding. All authors contributed to writing the final version of this paper. All authors read and approved the final manuscript.

\section{Ethics approval and consent to participate}

The Institutional Review Boards' approval of the study protocol was obtained from Wuhan Center for Disease Prevention and Control, Wuhan, China (reference number WHCDCIRB-K-2016024). Since the study used only the surveillance data collected by the Wuhan Center for Disease Prevention and Control, inform consent to participate was not required.

\section{Consent for publication}

Not applicable.

\section{Competing interests}

The authors declare that they have no competing interests.

\section{Publisher's Note}

Springer Nature remains neutral with regard to jurisdictional claims in published maps and institutional affiliations.

\section{Author details}

'Department of Infectious Diseases Control and Prevention, Wuhan Center for Disease Control and Prevention, Wuhan, Hubei, China. ${ }^{2}$ Department of Hygiene, Sapporo Medical University School of Medicine, S-1, W-17, Chuo-ku, Sapporo, Hokkaido 060-8556, Japan. ${ }^{3}$ Institute of Infectious Disease Control and Prevention, Hubei Center for Disease Control and Prevention, Wuhan, China. ${ }^{4}$ Wuhan Centers for Disease Control and Prevention, 24 Jianghanbei Road, Wuhan, Hubei 430000, China.

Received: 18 November 2016 Accepted: 26 July 2017

\section{Published online: 03 August 2017}

\section{References}

1. Adriana Lopez, Scott Schmid, Stephanie Bialek. Manual for the surveillance of vaccine-preventable diseases, chapter 17: Varicella. 2011, http://www.cdc. gov/vaccines/pubs/surv-manual/chpt17-varicella.html.

2. Council of State and Territorial Epidemiologists. Position statement 09-ID-68. Public health reporting and National Notification for Varicella: CSTE; 2009. http://c.ymcdn.com/sites/www.cste.org/resource/resmgr/PS/09-ID-68.pdf. Accessed 6 July 2017.

3. Harigane K, Sumi A, Mise K, Kobayashi N. The role of temperature in reported chickenpox cases from 2000 to 2011 in Japan. Epidemiol Infect. 2015;143:2666-78

4. Yang Y, Geng X, Liu X, Wang W, Zhang J. Association between the incidence of varicella and meteorological conditions in Jinan, eastern China, 2012-2014. BMC Infect Dis. 2016;16:179.

5. Breuer J, Fifer H. Chickenpox. BMJ Clin Evid. 2011;2011:0912.

6. Boelle PY, Hanslik T. Varicella in non-immune persons: incidence, hospitalization and mortality rates. Epidemiol Infect. 2002;129:599-606.

7. Wu PY, Li YC, Wu HD. Risk factors for chickenpox incidence in Taiwan from a large-scale computerized database. Int J Dermatol. 2007;46:362-6.

8. Chen B, Sumi A, Toyoda S, Hu Q, Zhou D, Mise K, Zhao J, Kobayashi N. Time series analysis of reported cases of hand, foot, and mouth disease from 2010 to 2013 in Wuhan, China. BMC Infect Dis. 2015;15:495-9.

9. Wnag L, Wang Y, Jin S, Wu Z, Chin DP, Koplan JP, Wilson ME. Emergence and control of infectious diseases in China. Lancet. 2008:372:1598-605

10. Gil A, San-Martin M, Carrasco P, Gonzalez A. Epidemiology of severe varicella-zoster virus infection in Spain. Vaccine. 2004;22:3947-51.

11. Marchetto S, de Benedictis FM, de Martino M, Versace A, Chiappini E, Bertaine C, Osimani P, Cordiali R, Gabiano C, Galli L. Epidemiology of hospital admissions for chickenpox in children: an Italian multicentre study in the pre-vaccine era. Acta Paediatr. 2007;96:1490-3.
12. JYC C, Lin HL, Tian LW. Meteorological factors and el Nino southern oscillation are associated with paediatric varicella infections in Hong Kong, 2004-2010. Epidemiol Infect. 2014;142:1384-92.

13. National Bureau of Statistics of China (http://www.stats.gov.cn/english).

14. The Hong Kong census and statistic department website (http://www. censtatd.gov.hk/home.html).

15. The Hong Kong observatory website (http://www.hko.gov.hk).

16. Department of Health. Chickenpox vaccine to incorporate into $\mathrm{DH}$ childhood immunisation programme on July 22014 (press release, 24 June 2014). In: Family Heath Service Department of Health, HKSAR government; 2014.

17. WWS T, Chan J, KKH L, Lee A, PKS C, Chan D, EAS N. Parental attitudes and factors associated with varicella vaccination in preschool and school children in Hong Kong. Medicine. 2015;94:1-8.

18. JYC C, Leung KM, WWS T, Lee A. Varicella vaccine uptake and asscoated fatocrs in children in Hong Kong. Epidemiol Infect. 2014;142:994-1001.

19. Lopez AS, Cardemil C, Pabst LJ, Cullen KA, Leung J, Bialek SR. Two-dose varicella vaccination coverage among children aged 7 years - six sentinel sites, United States, 2006-2012. MMWR. 2014;63:174-7.

20. Sumi A, Luo T, Zhou D, Yu B, Kong D, Kobatashi N. Time-series analysis of hepatitis $\mathrm{a}, \mathrm{B}, \mathrm{C}$ and $\mathrm{E}$ infections in a large Chinese city: application to prediction analysis. Epidemiol Infect. 2013;141:905-15.

21. Sumi A, Ohtomo N, Tanaka Y, Sawamura S, Olsen LF, Kobayashi N. Prediction analysis for measles epidemics. Jpn J Appl Phys. 2003;42:7611-20.

22. Ohtomo N, Kamo T, Watanabe M, Yoneyama K, Tanaka Y, Hayashi R. Power spectral densities of temporal variations of blood pressures. Jpn J Appl Phys. 1996:35:5571-82.

23. Ohtomo N, Sumi A, Tanaka Y, Tokiwano K, Terachi S. A detailed study of power spectral density for Rossler system. J Phys Soc Jpn. 1996;65:2811-23.

24. Terachi S, Ohtomo N, Tanaka Y. Quantitative evaluation of MemCalc Sysytem. In: Saito K, Koyama A, Yoneyama K, Sawada Y, Ohtomo N, editors. A recent advance in time series analysis by maximum entropy methodapplications to medical and biological sciences. Sapporo: Hokkaido University Press; 1994. p. 49-62.

25. Shoji M, Tsunoda A, Ishida N. Correlation between the occurrence of infantile infectious diseases and the weather. Journal of Tuberculosis and Leprosy. 1986;38:91-101. in Japanese

26. Brisson M, Edmunds WJ, Law B, Gay NJ, Walld R, Brownell M, et al. Epidemiology of varicella zoster virus infection in Canada and the United Kingdom. Epidemiol Infect. 2001:127:305-14.

27. JYC C, Tian L, Kwan YW, Chan WM, Leung CW. Hospitalisations for varicella among children and adolescents in a tertiary referral hospital in Hong Kong, 2004 to 2008. BMC Public Health. 2011;11:366-72.

28. Critselis E, Nastos PT, Theodoridou K, Theodoridou M, Tsolia MN, Hadjichristodoulou C, Papaevangelou V. Time trends in pediatric hospitalizations for varicella infections are associated with climatic changes: a 22-year retrospective study in a tertiary Greek referral center. PLoS One. 2012;7:e52016.

29. Ogura Y. Ippan Kisho-gaku. Tokyo: Tokyo University Press; 1984. in Japanese

30. Lee WB. Review of varicella zoster seroepidemiology in India and south-east Asia. Trop Med Int Health. 1998;3:886-90.

31. Garnett GP, et al. The age in infection with varicella zoster virus in St Lucia, West Indies. Epidemiol Infect. 1993;110:361-72.

32. White E. Chickenpox in Kerala. Indian J Public Health. 1978;22:141-51.

33. World Health Organization. Epidemiology of chickenpox 1997-1990. Weekly Epidemiology Record. 1992;67:118-9.

34. Rice PS. Ultra-violet radiation is responsible for the differences in global epidemiology of chickenpox and the evolution of varicella-zoster virus as man migrated out of Africa. Virol J. 2011:8:189-94.

35. Sumi A, Kamo K, Ohtomo N, Kobayashi N. Study of the effect of vaccination on periodic structures of measles epidemics in Japan. Microbiol Immunol. 2007:51:805-14

36. Souty C, Turbelin C, Blanchon T, Hanslik T, Strat RL, Boëlle PY. Improving disease incidence estimates in primary care surveillance systems. Popul Health Metrics. 2014;12:19-27. 